\title{
Homotopy Classification of Connected Sums of Sphere Bundles over Spheres, II
}

\author{
By \\ Hiroyasu Ishimoto*
}

\section{Introduction}

In the classification problems of manifolds, the connected sums of sphere bundles over spheres appear frequently. For example, we can see those in [6], [7], and [13]. Motivated by those, in the preceding paper [8], we classified the connected sums consisting of sphere bundles over spheres which admit crosssections up to homotopy equivalence.

In this paper, as promised previously, we investigate the general case. And, under some assumptions on dimensions, i.e. in metastable range, we obtain a necessary and sufficient condition for two connected sums of sphere bundles over spheres to be homotopy equivalent, by extending the results of JamesWhitehead [10] and using the handlebody theory of Wall [14] and Ishimoto [5]. Applications of the main theorem to special cases will appear in the subsequent paper.

Let $B_{i}, i=1,2, \cdots, r$, be $p$-sphere bundles over $q$-spheres $(p, q>1)$, and let $\bar{B}_{i}, i=1,2, \cdots, r$, be the associated $(p+1)$-disk bundles. It is understood that each $B_{i}$, or $\bar{B}_{i}$, also denotes the total space of the bundle and has the oriented differentiable structure induced from those of the fibre and the base space. If $p \geqq q$, each $B_{i}$ admits a cross-section, and the homotopy classification of the connected sums of such bundles has been completed in [8]. So, we assume that $p<q-1$. The torsion case that $p=q-1$ is excluded from this paper and the problem is still open. We denote the characteristic element of $B_{i}$ by $\alpha\left(B_{i}\right)$ or simply by $\alpha_{i}$ and we put $\varepsilon_{i}=\pi_{*}\left(\alpha_{i}\right)$, where $\pi_{*}: \pi_{q-1}\left(S O_{p+1}\right) \rightarrow \pi_{q-1}\left(S^{p}\right)$ is the homomorphism induced from the projection $\pi: S O_{p+1} \rightarrow S O_{p+1} / S O_{p}=S^{p}$.

The boundary connected sum $\forall_{i=1}^{r} \bar{B}_{i}$ can be considered as a handlebody of

Communicated by N. Shimada, February 27, 1981.

* Department of Mathematics, Kanazawa University, Kanazawa 920, Japan. 
$\mathscr{H}(m+1, r, q), m=p+q$, and the connected sum $\#_{i=1}^{r} B_{i}$ is its boundary. In general, $\#_{i=1}^{r} B_{i}$ may have various representations into the connected sums of $p$-sphere bundles over $q$-spheres up to diffeomorphism. In fact, we can observe it using the handlebody theory as follows.

Let $W$ be a handlebody of $\mathscr{H}(m+1, r, q), m=p+q$, and assume that $2 p>q>1$. Let $\phi: H \times H \rightarrow \pi_{q}\left(S^{p+1}\right), H=H_{q}(W)$, be the pairing defined by Wall [14], and let $\alpha: H \rightarrow \pi_{q-1}\left(S O_{p+1}\right)$ be the map assigning to each $x \in H$ $\cong \pi_{q}(W)$ the characteristic element of the normal bundle of the imbedded $q$ sphere which represents $x$. $\alpha$ is a quadratic form with the associated homomorphism $\partial \circ \phi$, where $\partial: \pi_{q}\left(S^{p+1}\right) \rightarrow \pi_{q-1}\left(S O_{p+1}\right)$ belongs to the homotopy exact sequence of the fibering $S O_{p+1} \rightarrow S O_{p+2} \rightarrow S^{p+1}$. ([14], p. 257). A base $\left\{w_{1}\right.$, $\left.w_{2}, \cdots, w_{r}\right\}$ of the free abelian group $H$ is called admissible if $\phi\left(w_{i}, w_{j}\right)=0$ for all $i, j(i \neq j)$. If $H$ has an admissible base $\left\{w_{1}, w_{2}, \cdots, w_{r}\right\}$, then $W$ can be represented as a boundary connected sum of $(p+1)$-disk bundles over $q$-spheres with the characteristic elements $\alpha\left(w_{i}\right), i=1,2, \cdots, r$. For, we can take the imbedded $q$-spheres which represent $w_{i}, i=1,2, \cdots, r$, to be disjoint (cf. Ishimoto [5]). Hence, by tying the tubular neighbourhoods of such imbedded $q$-spheres with thin bands in $W$, and by the $h$-cobordism theorem, we know that $W$ is diffeomorphic $(m>4)$ to such a boundary connected sum of disk bundles over spheres.

Thus, the representations of $W=q_{i=1}^{r} \bar{B}_{i}$ into the boundary connected sums of $(p+1)$-disk bundles over $q$-spheres correspond with the admissible bases of $H=H_{q}(W)$. Since $H_{q}(\partial W) \cong H_{q}(W)$ if $p \neq q-1$, $q$, we obtain various representations of $\partial W=\#_{i=1}^{r} B_{i}$ into the connected sums of $p$-sphere bundles over $q$ spheres associated with the admissible bases of $H \cong H_{q}(\partial W)$.

In Section 2, it is shown that Wall's pairing is a homotopy invariant of the boundary of the handlebody if $p \neq q-1$. That is,

Proposition 1. Let $W, W^{\prime}$ be handlebodies of $\mathscr{H}(p+q+1, r, q)$ and assume that $2 p>q>1$ and $p \neq q-1$. If there exists a homotopy equivalence $f: \partial W \rightarrow \partial W^{\prime}$ which preserves orientation, then for the isomorphism $h=i_{*}^{\prime} \circ f_{*}$ $\circ i_{*}^{-1}: H_{q}(W) \rightarrow H_{q}\left(W^{\prime}\right)$, we have $\phi=\phi^{\prime} \circ(h \times h)$, where $\phi, \phi^{\prime}$ are Wall's pairings of $W, W^{\prime}$ and $i, i^{\prime}$ are inclusion maps of $\partial W, \partial W^{\prime}$ into $W, W^{\prime}$, respectively.

If $p \geqq q$, the proposition is trivial since $\phi=\phi^{\prime}=0$. Hence, it makes sense for $p \leqq q-1$. Note that $\phi(x, x)=\left(E \circ \pi_{*}\right)(\alpha(x))$ by [14], where $E$ is the suspension homomorphism. Immediately we have the following.

Corollary 2. Under the above assumptions on $p, q$, if $\partial W$ has the homotopy 
type of $\sharp_{i=1}^{r} B_{i}$, a connected sum consisting of p-sphere bundles over q-spheres, then $W$ is represented into a boundary connected sum of $(p+1)$-disk bundles over $q$-spheres, and hence $\partial W$ into a connected sum of p-sphere bundles over $q$-spheres. Furthermore, if $s$ bundles in $B_{i}, i=1,2, \cdots, r$, admit cross-sections, $\partial W$ is represented into a connected sum of p-sphere bundles over $q$-spheres in which s bundles admit cross-sections.

Corollary 3. Under the above assumptions on $p, q$, if $H=H_{q}(W)$ has no admissible bases, then $\partial W$ never has the homotopy type of a connected sum of p-sphere bundles over $q$-spheres.

Let $\omega$ be an element of $\pi_{q-1}\left(S^{p}\right)$. We have the following homomorphisms

$$
\pi_{p+q-1}\left(S^{q-1}\right) \stackrel{\omega *}{\longrightarrow} \pi_{p+q-1}\left(S^{p}\right) \stackrel{J}{\longleftarrow} \pi_{q-1}\left(S O_{p}\right) \stackrel{i *}{\longrightarrow} \pi_{q-1}\left(S O_{p+1}\right),
$$

where $\omega_{*}$ is defined by the composition with $\omega, J$ is the $J$-homomorphism, and $i_{*}$ is induced from the inclusion. Let $G(\omega)=i_{*}\left(J^{-1}\left(\operatorname{Im} \omega_{*}\right)\right.$ ) (James-Whitehead [9]).

Let $H, \phi, \alpha$ and $\varepsilon=\pi_{*} \circ \alpha$ be the invariants of $W=\xi_{i=1}^{r} \bar{B}_{i}$, where $\varepsilon$ is a quadratic form with the associated homomorphism $\pi_{* \circ} \circ \circ \phi$. We note that if $p \neq q-1$, $q$, then $(H ; \phi, \alpha)$ is determined from $\partial W=\#_{i=1}^{r} B_{i}$. In fact, $H=H_{q}(W)$ $\cong H_{q}(\partial W), \alpha\left(w_{i}\right)=\alpha\left(B_{i}\right), i=1,2, \cdots, r$, where $\left\{w_{1}, \cdots, w_{r}\right\}$ is the canonical basis of $H$ represented by zero cross-sections of $\bar{B}_{l}, i=1,2, \cdots, r$, and $\phi\left(w_{i}, w_{j}\right)=0$ if $i \neq j, \phi\left(w_{i}, w_{i}\right)=E \pi_{*} \alpha\left(B_{i}\right)$ for each $i, j$. Let $B_{i}^{\prime}, i=1,2, \cdots, r^{\prime}$, be another set of $p$-sphere bundles over $q$-spheres $(p \neq q-1)$. If $\#_{i=1}^{r} B_{i}$ has the homotopy type of $\#_{i=1}^{\prime} B_{i}^{\prime}$, then $r=r^{\prime}$ by those homological aspect. Therefore, we assume that $r=r^{\prime}$ henceforth. Similarly define $H^{\prime}, \phi^{\prime}, \alpha^{\prime}$, and $\varepsilon^{\prime}$ for $W^{\prime}=\sharp_{i=1}^{r} \bar{B}_{i}^{\prime}$. Let $\alpha_{i}, \alpha_{i}^{\prime}$ be the characteristic elements of $B_{i}, B_{i}^{\prime}$ respectively and put $\varepsilon_{i}=\pi_{*}\left(\alpha_{i}\right)$, $\varepsilon_{i}^{\prime}=\pi_{*}\left(\alpha_{i}^{\prime}\right), i=1,2, \cdots, r$. We obtain the following.

Theorem 4. Let $q / 2<p<q-1$. Then, the connected sums $\#_{i=1}^{r} B_{i}, \#_{i=1}^{r} B_{i}^{\prime}$ are of the same oriented homotopy type if and only if $\varepsilon_{i}=\varepsilon_{i}^{\prime}$ and $\left\{\alpha_{i}\right\}=\left\{\alpha_{i}^{\prime}\right\}$ in $\pi_{q-1}\left(S O_{p+1}\right) / G\left(\varepsilon_{i}\right)=\pi_{q-1}\left(S O_{p+1}\right) / G\left(\varepsilon_{i}^{\prime}\right)$ for $i=1,2, \cdots, r$ "modulo representations". More precisely, they are of the same oriented homotopy type if and only if there exist the admissible bases $\left\{w_{1}, \cdots, w_{r}\right\},\left\{w_{1}^{\prime}, \cdots, w_{r}^{\prime}\right\}$ of $H, H^{\prime}$ respectively such that

(i) $\varepsilon\left(w_{i}\right)=\varepsilon^{\prime}\left(w_{i}^{\prime}\right), i=1,2, \cdots, r$, (i.e. $\left.\varepsilon \cong \varepsilon^{\prime}\right)$ and

(ii) $\left\{\alpha\left(w_{i}\right)\right\}=\left\{\alpha^{\prime}\left(w_{i}^{\prime}\right)\right\} \quad$ in $\pi_{q-1}\left(S O_{p+1}\right) / G\left(\varepsilon\left(w_{i}\right)\right)=\pi_{q-1}\left(S O_{p+1}\right) / G\left(\varepsilon^{\prime}\left(w_{i}^{\prime}\right)\right)$, $i=1,2, \cdots, r$. 
If all $B_{i}, B_{i}^{\prime}, i=1,2, \cdots, r$, admit cross-sections, then $\phi=\phi^{\prime}=0$. So, any bases of $H, H^{\prime}$ are admissible, $\alpha, \alpha^{\prime}$ are the homomorphisms, and $\varepsilon=\varepsilon^{\prime}=0$. Furthermore, $G(0)=i_{*} J^{-1}(0)$ induces $i_{*} \pi_{q-1}\left(S O_{p}\right) / G(0) \cong J \pi_{q-1}\left(S O_{p}\right) / P \pi_{q}\left(S^{p}\right)$, where $P=\left[, \iota_{p}\right]$ and $\iota_{p}$ is the orientation generator of $\pi_{p}\left(S^{p}\right)$ (cf. [10], p. 152). Hence, we have Theorem 1 of [8] for $p<q-1$.

Proposition 1 is proved in Section 2, and using it Theorem 4 is proved in Section 4 and Section 5.

\section{§1. Cell Structure and Linking Elements}

Let $W=D^{m+1} \cup_{\left\{\varphi_{i}\right\}}\left\{\cup_{i=1}^{r} D_{i}^{q} \times D_{i}^{p+1}\right\}$ be a handlebody of $\mathscr{H}(m+1, r, q)$, $m=p+q, p, q>1$, where $\varphi_{i}: \partial D_{i}^{q} \times D_{i}^{p+1} \rightarrow \partial D^{m+1}, i=1,2, \cdots, r$, are the disjoint imbeddings. Let $Y=S^{m}-\cup_{i=1}^{r} \operatorname{Int} \varphi_{i}\left(S_{i}^{q-1} \times D_{i}^{p+1}\right)$. Then $\partial W=$ $Y \cup_{\left\{\bar{\varphi}_{i}\right\}}\left\{\cup_{i=1}^{r} D_{i}^{q} \times S_{i}^{p}\right\}$, where $\bar{\varphi}_{i}=\varphi_{i} \mid S_{i}^{q-1} \times S_{i}^{p}, i=1,2, \cdots, r$. Let $\widetilde{S}_{i}^{p} \subset$ Int $Y$ be the imbedded $p$-sphere slightly moved from $x_{i} \times S_{i}^{p}, x_{i} \in \partial D_{i}^{q}$, where $i=1,2, \cdots, r$. We join $\tilde{S}_{i}^{p}, i=1,2, \cdots, r$, by $r$ arcs in Int $Y$ from a fixed point and take a thin closed neighbourhood $N . \quad N$ has the homotopy type of $\vee_{i=1}^{r} S_{i}^{p}$.

By the Alexander duality theorem, we have

$$
H_{i}(N) \cong H_{i}(Y) \quad \text { if } \quad i<m-1,
$$

and, since $N, Y$ are simply connected,

$$
\pi_{i}(N) \cong \pi_{i}(Y) \quad \text { if } \quad i<m-2,
$$

where the isomorphisms are induced from the inclusion map. So that, $H_{i}(Y, N)$ $\cong 0$ for $i<m-1$, and therefore by the homology exact sequence of $(\partial W, Y, N)$, we have

$$
H_{i}(\partial W, N) \cong H_{i}(\partial W, Y) \quad \text { if } \quad i<m-1 .
$$

Here, by the excision theorem,

$$
H_{i}(\partial W, Y) \cong \begin{cases}\mathbb{Z}+\cdots+\mathbb{Z} & \text { if } i=q, m \\ 0 & \text { otherwise, }\end{cases}
$$

and $\left[D_{i}^{q} \times y_{i}\right], y_{i} \in S_{i}^{p}, i=1,2, \cdots, r$, form a basis of $H_{q}(\partial W, Y)$. Hence, noting that $N, Y$ and $\partial W$ are simply connected and $H_{i}(\partial W, N) \cong H_{i}(\partial W, Y) \cong 0$ for $i<q$, we know

$$
\begin{aligned}
& \pi_{q}(\partial W, N) \cong H_{q}(\partial W, N), \\
& \pi_{q}(\partial W, Y) \cong H_{q}(\partial W, Y),
\end{aligned}
$$


by the Hurewicz isomorphism theorem.

Let $V=\partial W-\operatorname{Int} D^{m}$ and we may assume that $N \subset \operatorname{Int} V$. Then, by the homology exact sequence of $(\partial W, V, N)$,

$$
H_{i}(V, N) \cong H_{i}(\partial W, N) \quad \text { if } \quad i<m,
$$

and similarly as above,

$$
\pi_{q}(V, N) \cong H_{q}(V, N) .
$$

Thus, we have the following commutative diagram

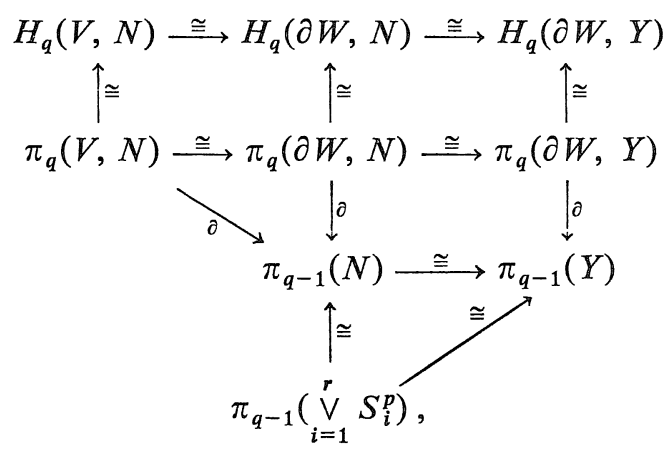

where the horizontal isomorphisms are all induced from the inclusion maps.

We note that $H_{m-1}(\partial W, N) \cong 0$ by the homology exact sequence of $(\partial W, N)$ and $H_{m}(V, N) \cong 0$. So that, $H_{i}(V, N) \cong 0$ if $i \neq q$. Let $m>5$. Then, by [12] or applying Theorem 7.6 and 7.8 of $[11]$ to the triad $\left(V^{\prime} ; \partial N, S^{m-1}\right)$, where $V^{\prime}=V$-Int $N$, we obtain the $q$-handles $T_{i}$ in $V^{\prime}, i=1,2, \cdots, r$, such that the homology classes $\left[T_{i}\right], i=1,2, \cdots, r$, form the basis of $H_{q}\left(V^{\prime}, \partial N\right) \cong H_{q}(V, N)$ which corresponds to the basis $\left\{\left[D_{i}^{q} \times y_{i}\right], i=1,2, \cdots, r\right\}$ of $H_{q}(\partial W, Y)$. We may identify $V$ with $N \cup T_{1} \cup T_{2} \cup \cdots \cup T_{r}$.

Henceforth, we assume that $2 p>q>1$ and $m>5$. Let $\lambda_{j}=\sum_{i=1}^{r} \lambda_{i j}$ $\in \pi_{q-1}\left(\vee_{i=1}^{r} S_{i}^{p}\right)=\sum_{i=1}^{r} \pi_{q-1}\left(S_{i}^{p}\right)$ be the linking element of the link $\left\{\cup_{i=1}^{r} \varphi_{i}\right.$ $\left.\left(S_{i}^{q-1} \times o\right)\right\} \cup \varphi_{j}\left(S_{j}^{q-1} \times y_{j}\right) \subset S^{m}$ defined by $\varphi_{j}\left(S_{j}^{q-1} \times y_{j}\right) \subset S^{m}-\cup_{i=1}^{r} \varphi_{i}\left(S_{i}^{q-1} \times o\right)$ $\simeq Y . \quad \lambda_{i j} \in \pi_{q-1}\left(S_{i}^{p}\right)$ coincides with the linking element of the link $\varphi_{i}\left(S_{i}^{q-1} \times o\right)$ $\cup \varphi_{j}\left(S_{j}^{q-1} \times o\right) \subset S^{m}$ defined by $\varphi_{j}\left(S_{j}^{q-1} \times o\right) \subset S^{m}-\varphi_{i}\left(S_{i}^{q-1} \times 0\right) \simeq S_{i}^{p}(i \neq j) . \quad \lambda_{j j}$ coincides with the linking element of the link $\varphi_{j}\left(S_{j}^{q-1} \times o\right) \cup \varphi_{j}\left(S_{j}^{q-1} \times y_{j}\right) \subset S^{m}$ and is called the self-linking element of $\varphi_{j}\left(S_{j}^{q-1} \times o\right)$. Note that $\lambda_{j j}=\pi_{*} \alpha\left(w_{j}\right)$, where $w_{j}$ is the basis element of $H_{q}(W) \cong H_{q}\left(W, D^{m+1}\right)$ determined by $\left[D_{j}^{q} \times o\right]$. Let $v_{j} \in \pi_{q-1}(Y)$ be the homotopy class of $\varphi_{j} \mid S_{j}^{q-1} \times y_{j}, j=1,2, \cdots, r$. Then, in the above diagram, $v_{j}$ corresponds to $\lambda_{j}$ for $j=1,2, \cdots, r$. Hence, by com- 
mutativity of the diagram, the attaching map of the $q$-axis of $T_{j}$ is given by $\lambda_{j}, j=1,2, \cdots, r$. Thus, we have

Lemma 1.1. Let $W=D^{m+1} \cup_{\left\{\varphi_{i}\right\}}\left\{\cup_{i=1}^{r} D_{i}^{q} \times D_{i}^{p+1}\right\}$ be a handlebody of $\mathscr{H}(m+1, r, q)$, where $m=p+q$ and $\varphi_{i}: \partial D_{i}^{q} \times D_{i}^{p+1} \rightarrow \partial D^{m+1}, i=1,2, \cdots, r$, are disjoint imbeddings. We assume that $2 p>q>1$ and $(p, q) \neq(2,3)$. Then, $\partial W$ has the homotopy type of

$$
\left(\bigvee_{i=1}^{r} S_{i}^{p}\right) \cup\left(\bigcup_{j=1}^{r} D_{j}^{q}\right) \cup D^{m}
$$

and the attaching map of each $D_{j}^{q}$ is given by $\lambda_{j}=\sum_{i=1}^{r} \lambda_{i j} \in \pi_{q-1}\left(\vee_{i=1}^{r} S_{i}^{p}\right)$ $=\sum_{i=1}^{r} \pi_{q-1}\left(S_{i}^{p}\right)$, where each $\lambda_{i j}$ is the linking element of the link $\varphi_{i}\left(S_{i}^{q-1} \times o\right)$ $\cup \varphi_{j}\left(S_{j}^{q-1} \times o\right) \subset S^{m}(i \neq j)$ and $\lambda_{j j}$ is the self-linking element of $\varphi_{j}\left(S_{j}^{q-1} \times o\right)$ $\subset S^{m}, i, j=1,2, \cdots, r$.

Remark. In each additional case for $m=4,5$, the lemma holds trivially since $\partial W$ is represented as a connected sum of $p$-sphere bundles over $q$-spheres which admit cross-sections.

\section{§ 2. Proof of Proposition 1}

Let $W, W^{\prime}$ be the handlebodies of $\mathscr{H}(m+1, r, q), m=p+q$, and assume that $1<p<q-1$ or $p>q>1$. Let $W^{\prime}=D^{\prime m+1} \cup_{\left\{\varphi_{i}^{\prime}\right\}}\left\{\cup_{i=1}^{r} D_{i}^{\prime q} \times D_{i}^{\prime p+1}\right\}$ be a representation, where $\varphi_{i}^{\prime}: \partial D_{i}^{\prime q} \times D_{i}^{\prime p+1} \rightarrow \partial D^{\prime m+1}, i=1,2, \cdots, r$, are disjoint imbeddings. By the assumption on $p, q$, we know that $H_{k}\left(\partial W^{\prime}\right) \cong 0$ if $k \neq 0, p, q$, $m, H_{p}\left(\partial W^{\prime}\right)$ has the basis $u_{i}^{\prime}=\left[x_{i}^{\prime} \times S_{i}^{\prime p}\right], i=1,2, \cdots, r$, and $H_{q}\left(\partial W^{\prime}\right)$ has the basis $v_{j}^{\prime}, j=1,2, \cdots, r$, which corresponds to $\left[D_{j}^{\prime q} \times o\right] \in H_{q}\left(W^{\prime}, D^{\prime m+1}\right), j=1$, $2, \cdots, r$, under the isomorphisms induced from the inclusion maps $H_{q}\left(\partial W^{\prime}\right)$ $\cong H_{q}\left(W^{\prime}\right) \cong H_{q}\left(W^{\prime}, D^{\prime m+1}\right)$. We call $\left\{u_{1}^{\prime}, \cdots, u_{r}^{\prime}\right\},\left\{v_{1}^{\prime}, \cdots, v_{r}^{\prime}\right\}$ to be the bases associated with the handles of $W^{\prime}$.

Lemma 2.1. For any homotopy equivalence $f: \partial W \rightarrow \partial W^{\prime}$ which preserves orientation, there exists a representation $W=D^{m+1} \cup_{\left\{\varphi_{i}\right\}}\left\{\cup_{i=1}^{r} D_{i}^{q} \times D_{i}^{p+1}\right\}$, where $\varphi_{i}: \partial D_{i}^{q} \times D_{i}^{p+1} \rightarrow \partial D^{m+1}, i=1,2, \cdots, r$, are disjoint imbeddings, such that $f_{*}\left(u_{i}\right)=u_{i}^{\prime}, f_{*}\left(v_{j}\right)=v_{j}^{\prime}$ for $i, j=1,2, \cdots, r$. Here, $\left\{u_{1}, \cdots, u_{r}\right\},\left\{v_{1}, \cdots, v_{r}\right\}$ are the bases of $H_{p}(\partial W), H_{q}(\partial W)$ respectively associated with the handles of $W$.

Proof. Let $\tilde{u}_{i}=f_{*}^{-1}\left(u_{i}^{\prime}\right), \tilde{v}_{j}=f_{*}^{-1}\left(v_{j}^{\prime}\right), i, j=1,2, \cdots, r$, and let $\tilde{w}_{j}=i_{*}\left(\tilde{v}_{j}\right)$, $j=1,2, \cdots, r$, where $i_{*}: H_{q}(\partial W) \cong H_{q}(W)$ is induced from the inclusion map. 
We represent $W$ by the basis $\left\{\tilde{w}_{1}, \cdots, \tilde{w}_{r}\right\}$ (cf. Milnor [11], Theorem 7.6). So, we have a representation $W=D^{m+1} \cup_{\left\{\varphi_{t}\right\}}\left\{\cup_{i=1}^{r} D_{i}^{q} \times D_{i}^{p+1}\right\}$. Then, clearly $i_{*}\left(v_{j}\right)=\tilde{w}_{j}=i_{*}\left(\tilde{v}_{j}\right)$ and therefore $\tilde{v}_{j}=v_{j}, j=1,2, \cdots, r$. Furthermore, $\tilde{u}_{i} \cdot \tilde{v}_{j}=\delta_{i j}$, $i, j=1,2, \cdots, r$, and $u_{i} \cdot \tilde{v}_{j}=\partial\left[x_{i} \times D_{i}^{p+1}\right] \cdot \tilde{v}_{j}=\left[x_{i} \times D_{i}^{p+1}\right] \cdot\left(i_{*}\left(\tilde{v}_{j}\right)\right)=\left[x_{i} \times D_{i}^{p+1}\right]$. $\tilde{w}_{j}=\delta_{i j}, i, j=1,2, \cdots, r$. Hence, $\tilde{u}_{i}=u_{i}, i=1,2, \cdots, r$.

Now, we prove Proposition 1. If $p \geqq q$, the assertion holds trivially. So, we assume that $2 p>q>1$ and $p<q-1$. Let $f: \partial W \rightarrow \partial W^{\prime}$ be a homotopy equivalence which preserves orientation. Let $W=D^{m+1} \cup_{\left\{\varphi_{i}\right\}}\left\{\cup_{i=1}^{r} D_{i}^{q} \times D_{i}^{p+1}\right\}$ be the representation given by Lemma 2.1. Then, by Lemma 1.1, we have the following diagram commutative up to homotopy.

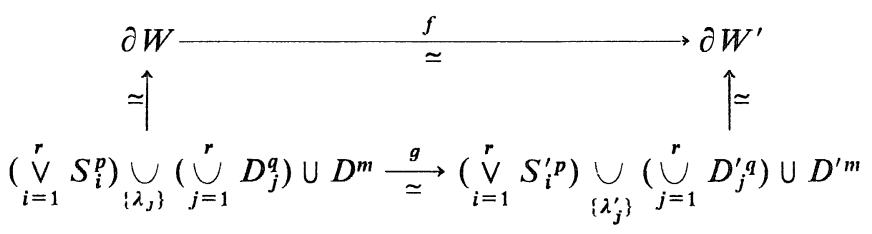

It may be assumed that $g\left(\left(\vee_{i=1}^{r} S_{i}^{p}\right) \cup_{\left\{\lambda_{j}\right\}}\left(\cup_{j=1}^{r} D_{j}^{q}\right)\right) \subset\left(\vee_{i=1}^{r} S_{i}^{\prime p}\right) \cup_{\left\{\lambda_{j}^{\prime}\right\}}\left(\cup_{j=1}^{r} D_{i}^{\prime q}\right)$ and each $g \mid S_{i}^{p}$ is the identity $\left(S_{i}^{p}, S_{i}^{\prime}\right.$ are copies of $\left.S^{p}\right)$ since $f_{*}\left(u_{i}\right)=u_{i}^{\prime}, i=1$, $2, \cdots, r$. Hence, we have the following commutative diagram, where we put $X=\left(\vee_{i=1}^{r} S_{i}^{p}\right) \cup_{\left\{\lambda_{j}\right\}}\left(\cup_{j=1}^{r} D_{j}^{q}\right) \cup D^{m}$ and $X^{\prime}=\left(\vee_{i=1}^{r} S_{i}^{\prime p}\right) \cup_{\left\{\lambda_{j}^{\prime}\right\}}\left(\cup_{j=1}^{r_{j}} D_{j}^{\prime q}\right) \cup$ $D^{\prime m}$.

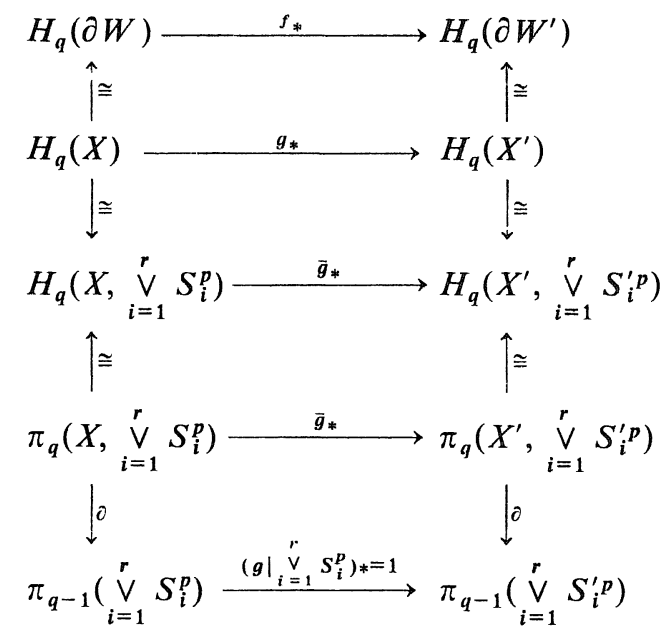

Note that each $v_{j}, v_{j}^{\prime}$ correspond to $\left[D_{j}^{q}\right] \in H_{q}\left(X, \vee_{i=1}^{r} S_{i}^{p}\right),\left[D_{j}^{\prime q}\right] \in H_{q}\left(X^{\prime}\right.$, $\left.\vee_{i=1}^{r} S_{i}^{\prime q}\right)$ respectively. $\left\{D_{j}^{q}\right\} \in \pi_{q}\left(X, \vee_{i=1}^{r} S_{i}^{p}\right),\left\{D_{j}^{\prime q}\right\} \in \pi_{q}\left(X^{\prime}, \vee_{i=1}^{r} S_{i}^{p}\right)$ correspond to $\left[D_{j}^{q}\right],\left[D_{j}^{\prime q}\right]$ under the Hurewicz isomorphisms. Then, since $f_{*}\left(v_{i}\right)$ 
$=v_{j}^{\prime}, j=1,2, \cdots, r$, we know that $\lambda_{j}=\partial\left\{D_{j}^{q}\right\}=\partial \bar{g}_{*}\left\{D_{j}^{q}\right\}=\partial\left\{D_{j}^{\prime q}\right\}=\lambda_{j}^{\prime}, j=1,2, \cdots$, $r$. So that, $\lambda_{i j}=\lambda_{i j}^{\prime}$ for all $i, j=1,2, \cdots, r$. On the other hand, $E \lambda_{i j}=\phi\left(w_{i}, w_{j}\right)$, $E \lambda_{i j}^{\prime}=\phi^{\prime}\left(w_{i}^{\prime}, w_{j}^{\prime}\right)$ by Lemma 7 of Wall [14], where $E$ is the suspension homomorphism, $w_{j}=i_{*}\left(v_{j}\right), w_{j}^{\prime}=i_{*}^{\prime}\left(v_{j}^{\prime}\right), j=1,2, \cdots, r$, and $i_{*}, i_{*}^{\prime}$ are the isomorphisms induced from inclusion maps. Therefore, $\phi\left(w_{i}, w_{j}\right)=\phi^{\prime}\left(w_{i}^{\prime}, w_{j}^{\prime}\right), i, j=1,2, \cdots, r$, and this yields $\phi\left(w_{i}, w_{j}\right)=\phi^{\prime} \circ(h \times h)\left(w_{i}, w_{j}\right), i, j=1,2, \cdots, r$, where $h=i_{*}^{\prime} \circ f_{*} \circ i_{*}^{-1}$. This completes the proof.

Let $B_{i}, B_{i}^{\prime}, i=1,2, \cdots, r$, be $p$-sphere bundles over $q$-spheres $(p, q>1)$ with the characteristic elements $\alpha_{i}, \alpha_{i}^{\prime}, i=1,2, \cdots, r$, respectively. Then, $\#_{i=1}^{r} B_{i}$ has the homotopy type of $X=\left(\vee_{i=1}^{r} S_{i}^{p}\right) \cup\left(\cup_{i=1}^{r} D_{i}^{q}\right) \cup D^{p+q}$, and $\#_{i=1}^{r} B_{i}^{\prime}$ the homotopy type of $X^{\prime}=\left(\vee_{i=1}^{r} S_{i}^{p}\right) \cup\left(\cup_{i=1}^{r} D_{i}^{\prime q}\right) \cup D^{p+q}$, where each $D_{i}^{q}, D_{i}^{\prime q}$ are attached to $S_{i}^{p}, S_{i}^{\prime p}$ by $\varepsilon_{i}=\pi_{*} \alpha_{i}, \varepsilon_{i}^{\prime}=\pi_{*} \alpha_{i}^{\prime}$ respectively (cf. [8] $\S 1$ ). Let $p<q-1$. Let $\left\{u_{i} ; i=1,2, \cdots, r\right\}$ be the basis of $H_{p}\left(\#_{i=1}^{r} B_{i}\right)$ represented by the fibres of $B_{i}$, $i=1,2, \cdots, r$. Since $H_{q}\left(\#_{i=1}^{r} B_{i}\right) \cong H_{q}\left(\#_{i=1}^{r} \bar{B}_{i}\right)$, the zero cross-sections of $\bar{B}_{i}$, $i=1,2, \cdots, r$, determine the basis $\left\{v_{1}, \cdots, v_{r}\right\}$ of $H_{q}\left(\#_{i=1}^{r} B_{i}\right) . \quad u_{i}$ corresponds to $\left[S_{i}^{p}\right] \in H_{p}(X)$ and $v_{i}$ to $\left[D_{i}^{q}\right] \in H_{q}(X), i=1,2, \cdots, r$. Those are the bases associated with handles if we consider $\#_{i=1}^{r} \bar{B}_{i}$ to be a handlebody. Similarly define $\left\{u_{i}^{\prime} ; i=1,2, \cdots, r\right\},\left\{v_{i}^{\prime} ; i=1,2, \cdots, r\right\}$ for $\#_{i=1}^{r} B_{i}^{\prime}$. Then, the above diagram and a similar argument will show the following, where $\pi_{q-1}\left(S_{i}^{p}\right)$, $\pi_{q-1}\left(S_{i}^{\prime p}\right)$ are direct summands of $\pi_{q-1}\left(\vee_{i=1}^{r} S_{i}^{p}\right), \pi_{q-1}\left(\vee_{i=1}^{r} S_{i}^{p}\right)$ respectively, $i=1,2, \cdots, r$.

Lemma 2.2. Let $1<p<q-1$. If there exists a map $f: \sharp_{i=1}^{r} B_{i} \rightarrow \#_{i=1}^{r} B_{i}^{\prime}$ such that $f_{*}\left(u_{i}\right)=u_{i}^{\prime}, f_{*}\left(v_{j}\right)=v_{j}^{\prime}, i, j=1,2, \cdots, r$, then $\varepsilon_{i}=\varepsilon_{i}^{\prime}$ for $i=1,2, \cdots, r$. Here, if $p \geqq q$ the assertion is trivial.

\section{§3. Difference of Bundles}

Let $B_{i}, B_{i}^{\prime}$ be $p$-sphere bundles over $q$-spheres with the characteristic elements $\alpha_{i}, \alpha_{i}^{\prime}$ respectively, $i=1,2, \cdots, r$, and assume that $\varepsilon_{i}=\varepsilon_{i}^{\prime}$, where $\varepsilon_{i}$ $=\pi_{*}\left(\alpha_{i}\right), \varepsilon_{i}^{\prime}=\pi_{*}\left(\alpha_{i}^{\prime}\right)$. Let $S_{i}^{p}, p_{i}$, and $S_{i}^{q}$ be respectively the fixed fibre, the projection, and the base space of $B_{i}$. Define $S_{i}^{\prime p}, p_{i}^{\prime}$, and $S_{i}^{\prime q}$ similarly for $B_{i}^{\prime}$. In the disjoint union of $B_{i}$ and $B_{i}^{\prime}$, identify $S_{i}^{p}$ with $S_{i}^{\prime p}$. Then, we have a $p$-sphere bundle over $S_{i}^{q} \vee S_{i}^{\prime q}$, where $S_{i}^{q}, S_{i}^{\prime q}$ are identified at $s_{i}=p_{i}\left(S_{i}^{p}\right)=p_{i}^{\prime}\left(S_{i}^{\prime p}\right)$. Since $B_{i}, B_{i}^{\prime}$ are included in this bundle as subspaces, we may denote it by $B_{i} \cup B_{i}^{\prime}$ (cf. [10], p. 156). 
Let $g_{i}: S^{q} \rightarrow S_{i}^{q} \vee S_{i}^{q q}$ be a map representing $\iota_{q}^{i}-\iota_{q}^{\prime i} \in \pi_{q}\left(S_{i}^{q} \vee S_{i}^{\prime q}\right)$, where $\iota_{q}^{i}, \iota_{q}^{\prime i}$ are the orientation generators of $\pi_{q}\left(S_{i}^{q}\right), \pi_{q}\left(S_{i}^{\prime q}\right)$ respectively. The induced bundle $A_{i}=g_{i}^{*}\left(B_{i} \cup B_{i}^{\prime}\right)$ has the characteristic element $\alpha_{i}-\alpha_{i}^{\prime}$ and admits a cross-section since $\pi_{*}\left(\alpha_{i}-\alpha_{i}^{\prime}\right)=\pi_{*}\left(\alpha_{i}\right)-\pi_{*}\left(\alpha_{i}^{\prime}\right)=\varepsilon_{i}-\varepsilon_{i}^{\prime}=0$ by the above assumption. Let $h_{\imath}: A_{\imath} \rightarrow B_{i} \cup B_{i}^{\prime}$ be the bundle map which covers $g_{i}$. A fixed fibre $S_{A_{i}}^{p}$ of $A_{i}$ is oriented so that $h_{i} \mid S_{A_{i}}^{p}: S_{A_{i}}^{p} \rightarrow S_{i}^{p}=S_{i}^{\prime p}$ is of degree 1 , and $A_{i}$ is oriented by the orientations of $S_{A_{i}}^{p}$ and $S^{q}$.

Let $S_{A_{i}}^{q}$ be the cross-section of $A_{i}$ associated with $\xi_{i} \in \pi_{q-1}\left(S O_{p}\right)$ satisfying $i_{*}\left(\xi_{i}\right)=\alpha_{i}-\alpha_{i}^{\prime}$. Then, $A_{i}=\left(S_{A_{i}}^{p} \vee S_{A_{i}}^{q}\right) \cup e_{A_{i}}^{p+q}$ and the attaching map is given by $\partial \tau_{i}=\iota_{p}^{A_{i} \circ \eta_{i}}+\left[\iota_{q}^{A_{i}}, \iota_{p}^{A_{i}}\right]$, where $\eta_{i}=J \xi_{i}$ and $\tau_{i}$ is the orientation generator of $\pi_{p+q}\left(A_{i}, S_{A_{i}}^{p} \vee S_{A_{i}}^{q}\right)$ (cf. [9]). Hence, by Lemma 1.1 of [8],

$$
\underset{i=1}{\#} A_{i} \simeq A=\left\{\bigvee_{i=1}^{r}\left(S_{A_{i}}^{p} \vee S_{A_{i}}^{q}\right)\right\} \cup e_{A_{i}}^{p+q},
$$

and the attaching map of the $(p+q)$-cell is given by

$$
\partial \tau=\sum_{i=1}^{r}\left(\iota_{p}^{A_{2} \circ \eta_{i}}+\left[\ell_{q}^{A_{i}}, \ell_{p}^{A_{i}}\right]\right),
$$

where $\tau$ is the orientation generator of $\pi_{p+q}\left(A, \vee_{i=1}^{r}\left(S_{A_{i}}^{p} \vee S_{A_{i}}^{q}\right)\right)$ and $\pi_{p+q-1}\left(S_{A_{i}}^{p} \vee S_{A_{i}}^{q}\right), i=1,2, \cdots, r$, are considered as direct summands of $\pi_{p+q-1}\left(\vee_{i=1}^{r}\left(S_{A_{i}}^{p} \vee S_{A_{i}}^{q}\right)\right)$.

In $A_{1} \# A_{2} \# \cdots \# A_{r}$, join every $(p+q-1)$-sphere where connected sum is performed to the base points of the bundles neighboring at the $(p+q-1)$-sphere by suitably chosen arcs. If we crush the $(p+q-1)$-spheres and the arcs to a point, the yielding space can be considered as $\vee_{i=1}^{r} A_{i}$. Let $v: \#_{i=1}^{r} A_{i} \rightarrow \vee_{i=1}^{r} A_{i}$ be the collapsing map. Then, we have a map

$$
h=\left(\bigvee_{i=1}^{r} h_{i}\right) \circ v: \prod_{i=1}^{r} A_{i} \longrightarrow \bigvee_{i=1}^{r}\left(B_{i} \cup B_{i}^{\prime}\right) .
$$

$\#_{i=1}^{r} A_{i}$ can be replaced by the complex $A$ of (3.1) and $h$ may be assumed to preserve the base point. We denote the map by the same symbol $h$.

$B_{i}$ has the cell structure $B_{i}=S_{i}^{p} \cup e_{i}^{q} \cup e_{i}^{p+q}$, where $e_{i}^{q}$ is attached to $S_{i}^{p}$ by $c_{p}^{i} \circ \varepsilon_{i}$. Here, $\iota_{p}^{i}$ is the orientation generator of $\pi_{p}\left(S_{i}^{p}\right)$. Let $\sigma_{i}$ be the orientation generator of $\pi_{p+q}\left(B_{i}, S_{i}^{p} \cup e_{i}^{q}\right)$. Then, $\partial \sigma_{\imath} \in \pi_{p+q-1}\left(S_{i}^{p} \cup e_{i}^{q}\right)$ is represented by the attaching map of $e_{i}^{p+q}$. Similarly to Lemma 1.1 of [8], it is seen that

$$
\underset{i=1}{\prod} B_{i} \simeq B=\left\{\bigvee_{i=1}^{r}\left(S_{i}^{p} \cup e_{i}^{q}\right)\right\} \cup e^{p+q},
$$

where each $e_{i}^{q}$ is attached by $\iota_{p}^{i} \circ \varepsilon_{i}$, and 


$$
\partial \sigma=\partial \sigma_{1}+\partial \sigma_{2}+\cdots+\partial \sigma_{r},
$$

where $\sigma$ is the orientation generator of $\pi_{p+q}\left(B, \vee{ }_{i=1}^{r}\left(S_{i}^{p} \cup e_{i}^{q}\right)\right)$ and each $\pi_{p+q-1}\left(S_{i}^{p} \cup e_{i}^{q}\right)$ is considered as a direct summand of $\pi_{p+q-1}\left(\vee_{i=1}^{r}\left(S_{i}^{p} \cup e_{i}^{q}\right)\right)$. Let $B_{i}^{\prime}=S_{i}^{\prime p} \cup e_{i}^{q} \cup e_{i}^{p+q}$ be the cell structure of $B_{i}^{\prime}$. $e_{i}^{q}{ }^{q}$ is attached to $S_{i}^{\prime p}$ by $\iota_{p}^{\prime i} \circ \varepsilon_{i}^{\prime}$, where $\iota_{p}^{\prime i}$ is the orientation generator of $\pi_{p}\left(S_{i}^{\prime p}\right)$. Let $\sigma_{i}^{\prime}$ be the orientation generator of $\pi_{p+q}\left(B_{i}^{\prime}, S_{i}^{\prime p} \cup e_{i}^{q} q\right)$.

Let $K=\vee_{i=1}^{r}\left(S_{i}^{p} \cup e_{i}^{q} \cup e_{i}^{\prime q}\right)$ be the subcomplex of $\vee_{i=1}^{r}\left(B_{i} \cup B_{i}^{\prime}\right)$, where $e_{i}^{q}, e_{i}^{\prime q}$ are attached to $S_{i}^{p}=S_{i}^{\prime p}$ by $\iota_{p}^{i} \circ \varepsilon_{i}$ and $\iota_{p}^{\prime i} \circ \varepsilon_{i}^{\prime}$ respectively. Then, it may be assumed that $h\left(\vee_{i=1}^{r}\left(S_{A_{i}}^{p} \vee S_{A_{i}}^{q}\right)\right) \subset K$ for the map $h: A \rightarrow \vee_{i=1}^{r}\left(B_{i} \cup B_{i}^{\prime}\right)$. Let $\bar{h}:\left(A, \vee r_{i=1}^{r}\left(S_{A_{i}}^{p} \vee S_{A_{i}}^{q}\right)\right) \rightarrow\left(\vee_{i=1}^{r}\left(B_{i} \cup B_{i}^{\prime}\right), K\right)$. From the construction of $h$, we know

$$
\bar{h}_{*}(\tau)=\left(\bar{\sigma}_{1}-\bar{\sigma}_{1}^{\prime}\right)+\left(\bar{\sigma}_{2}-\bar{\sigma}_{2}^{\prime}\right)+\cdots+\left(\bar{\sigma}_{r}-\bar{\sigma}_{r}^{\prime}\right),
$$

where $\bar{\sigma}_{i}$ is the image of $\sigma_{i}$ by the homomorphism induced from the inclusion $\left(B_{i}, S_{i}^{p} \cup e_{i}^{q}\right) \subset\left(B_{i} \cup B_{i}^{\prime}, S_{i}^{p} \cup e_{i}^{q} \cup e_{i}^{q}\right), i=1,2, \cdots, r, \bar{\sigma}_{i}^{\prime}$ is similar, and $\pi_{p+q}\left(B_{i} \cup B_{i}^{\prime}\right.$, $\left.S_{i}^{p} \cup e_{i}^{q} \cup e_{i}^{q}\right), i=1,2, \cdots, r$, are considered as the direct summands of $\pi_{p+q}\left(\vee{ }_{i=1}^{r}\left(B_{i} \cup B_{i}^{\prime}\right), K\right)$. Let $\delta_{i}=\partial \sigma_{i}$, and let $\bar{\delta}_{i}$ be the image of $\delta_{i}$ by the homomorphism induced from the inclusion $S_{i}^{p} \cup e_{1}^{q} \subset S_{i}^{p} \cup e_{i}^{q} \cup e_{i}^{\prime q}, i=1,2, \cdots, r$. Define $\delta_{i}^{\prime}, \bar{\delta}_{i}^{\prime}$ similarly, $i=1,2, \cdots, r$. Here, $\pi_{p+q-1}\left(S_{i}^{p} \cup e_{i}^{q} \cup e_{i}^{\prime q}\right), i=1,2, \cdots, r$, are understood as direct summands of $\pi_{p+q-1}\left(\vee_{i=1}^{r}\left(S_{i}^{p} \cup e_{i}^{q} \cup e_{i}^{\prime q}\right)\right)$. Then,

$$
\partial \bar{h}_{*} \tau=\partial \sum_{i=1}^{r}\left(\bar{\sigma}_{i}-\bar{\sigma}_{i}^{\prime}\right)=\sum_{i=1}^{r}\left(\partial \bar{\sigma}_{i}-\partial \bar{\sigma}_{i}^{\prime}\right)=\sum_{i=1}^{r}\left(\bar{\delta}_{i}-\bar{\delta}_{i}^{\prime}\right)=\sum_{i=1}^{r} \bar{\delta}_{i}-\sum_{i=1}^{r} \bar{\delta}_{i}^{\prime},
$$

and by (3.2),

$$
\partial \bar{h}_{*} \tau=h_{*} \partial \tau=\sum_{i=1}^{r} h_{*}\left(\iota_{p}^{A_{i} \circ} \eta_{i}+\left[\iota_{q}^{A_{i}}, \iota_{p}^{A_{i}}\right]\right)
$$

Hence, we have

$$
\sum_{i=1}^{r} \bar{\delta}_{i}-\sum_{i=1}^{r} \bar{\delta}_{i}^{\prime}=\sum_{i=1}^{r} h_{*}\left(c_{p}^{A_{i} \circ} \eta_{i}+\left[\zeta_{q}^{A_{i}}, \iota_{p}^{A_{i}}\right]\right) .
$$

\section{§4. Proof of the Necessity for Theorem 4}

Let $B_{i}, B_{i}^{\prime}, i=1,2, \cdots, r$, be $p$-sphere bundles over $q$-spheres with the characteristic elements $\alpha_{i}, \alpha_{i}^{\prime}$ respectively and assume that $q / 2<p<q-1$. Let $f: \#_{i=1}^{r} B_{i} \rightarrow \#_{i=1}^{r} B_{i}^{\prime}$ be a homotopy equivalence which preserves orientation.

Assertion 1. There exists another expression of $\sharp_{i=1}^{r} B_{i}$ into a connected 
sum of $p$-sphere bundles over q-spheres $\#_{i=1}^{r} \widetilde{B}_{i}$ such that in the cell decompositions $\#_{i=1}^{r} \widetilde{B}_{i} \simeq \widetilde{B}=\left\{\vee{ }_{i=1}^{r}\left(\widetilde{S}_{i}^{p} \cup \tilde{e}_{i}^{q}\right)\right\} \cup \tilde{e}^{p+q}$ and $\#_{i=1}^{r} B_{i}^{\prime} \simeq B=\left\{\vee{ }_{i=1}^{r}\left(S_{i}^{\prime p} \cup e_{i}^{\prime q}\right)\right\}$ $\cup e^{p+q}, f_{*}: H_{*}(\widetilde{B}) \rightarrow H_{*}\left(B^{\prime}\right)$ satisfies $f_{*}\left(\left[\widetilde{S}_{i}^{p}\right]\right)=\left[S_{i}^{p}\right], i=1,2, \cdots, r$ and $\bar{f}_{*}\left(\left[\tilde{e}_{j}^{q}\right]\right)$ $=\left[e_{j}^{\prime q}\right], j=1,2, \cdots, r$, where $f$ may be assumed to satisfy $f\left(\vee_{i=1}^{r} \tilde{S}_{i}^{p}\right) \subset \vee_{i=1}^{r} S_{i}^{p}$ and $\bar{f}:\left(\widetilde{B}, \vee{ }_{i=1}^{r} \tilde{S}_{i}^{p}\right) \rightarrow\left(B^{\prime}, \vee r_{i=1}^{r} S_{i}^{p}\right)$ is the relativization of $f$.

Proof. Let $W=\xi_{i=1}^{r} \bar{B}_{i}, W^{\prime}=\xi_{i=1}^{r} \bar{B}_{i}^{\prime}$, and let $\left\{u_{1}^{\prime}, \cdots, u_{r}^{\prime}\right\},\left\{v_{1}^{\prime}, \cdots, v_{r}^{\prime}\right\}$ be the bases of $H_{p}\left(\partial W^{\prime}\right), H_{q}\left(\partial W^{\prime}\right)$ respectively associated with the handles of $W^{\prime}$. Then, by Lemma 2.1 , there exists a representation of $W$ into such a handlebody that $f_{*}\left(u_{i}\right)=u_{i}^{\prime}, f_{*}\left(v_{j}\right)=v_{j}^{\prime}, i, j=1,2, \cdots, r$, where $\left\{u_{1}, \cdots, u_{r}\right\},\left\{v_{1}, \cdots, v_{r}\right\}$ are bases of $H_{p}(\partial W), H_{q}(\partial W)$ respectively associated with the new handles of $W$. Of course, $\left\{w_{j}^{\prime}=i_{*}^{\prime} v_{j}^{\prime} ; j=1,2, \cdots, r\right\}$, the basis of $H_{q}\left(W^{\prime}\right)$ is admissible since $w_{j}^{\prime}, j=1,2, \cdots, r$, are represented by zero cross-sections of $\bar{B}_{j}^{\prime}, j=1,2, \cdots, r$. Hence, by Proposition 1, the basis of $H_{q}(W),\left\{w_{j}=i_{*} v_{j} ; j=1,2, \cdots, r\right\}$ is admissible. Therefore, again $W$ can be represented into a boundary connected sum of $(p+1)$-disk bundles over $q$-spheres $\sharp_{i=1}^{r} \widetilde{B}_{i}$ and $\partial W$ into a connected sum of $p$-sphere bundles over $q$-spheres $\#_{i=1}^{r} \widetilde{B}_{i}$. We note that in the above celldecompositions, $u_{i}, v_{j}, u_{i}^{\prime}$, and $v_{j}^{\prime}$ correspond to $\left[\tilde{S}_{i}^{p}\right],\left[\tilde{e}_{j}^{q}\right],\left[S_{i}^{\prime p}\right]$, and $\left[e_{j}^{\prime q}\right]$ respectively for each $i, j$. This completes the proof.

Assertion 2. Under the cell decompositions $\#_{i=1}^{r} B_{i} \simeq B=\left\{\vee_{i=1}^{r}\left(S_{i}^{p} \cup e_{i}^{q}\right)\right\}$ $\cup e^{p+q}$ and $\#_{i=1}^{r} B_{i}^{\prime} \simeq B^{\prime}=\left\{\vee r_{i=1}^{r}\left(S_{i}^{\prime p} \cup e_{i}^{\prime q}\right)\right\} \cup e^{p+q}$, if $f_{*}: H_{*}(B) \rightarrow H_{*}\left(B^{\prime}\right)$ satisfies $f_{*}\left(\left[S_{i}^{p}\right]\right)=\left[S_{i}^{\prime p}\right], \bar{f}_{*}\left(\left[e_{j}^{q}\right]\right)=\left[e_{j}^{q}\right]$ for $i, j=1,2, \cdots, r$, then $\varepsilon_{i}=\varepsilon_{i}^{\prime}$, $i=1,2, \cdots, r$, where $\varepsilon_{i}=\pi_{*} \alpha_{i}, \varepsilon_{i}^{\prime}=\pi_{*} \alpha_{i}^{\prime}, \quad$ and $\left\{\alpha_{i}\right\}=\left\{\alpha_{i}^{\prime}\right\}, i=1,2, \cdots, r$, in $\pi_{q-1}\left(S O_{p+1}\right) / G\left(\varepsilon_{i}\right)=\pi_{q-1}\left(S O_{p+1}\right) / G\left(\varepsilon_{i}^{\prime}\right)$.

Proof. The former half of the assertion is known immediately from Lemma 2.2. To prove the latter half, we apply Section 3. By the assumption, we may assume that $f$ maps each $S_{i}^{p}$ identically onto $S_{i}^{\prime p}\left(S_{i}^{p}\right.$ and $S_{i}^{\prime p}$ can be identified by means of $\left.\epsilon_{p}^{\prime i} \circ\left(c_{p}^{i}\right)^{-1}\right)$ and $f\left(\vee_{i=1}^{r}\left(S_{i}^{p} \cup e_{i}^{q}\right)\right) \subset \vee{ }_{i=1}^{r}\left(S_{i}^{\prime p} \cup e_{i}^{\prime q}\right)$. Let $f^{0}=f \mid \vee{ }_{i=1}^{r}\left(S_{i}^{p} \cup e_{i}^{q}\right)$ and let $\rho: K \rightarrow \vee_{i=1}^{r}\left(S_{i}^{\prime p} \cup e_{i}^{\prime q}\right)$ be the retraction defined by $\rho \mid \vee r_{i=1}^{r}\left(S_{i}^{p} \cup e_{i}^{q}\right)=f^{0}$ and $\rho \mid \vee r_{i=1}^{r}\left(S_{i}^{p} \cup e_{i}^{\prime q}\right)=$ identity. Then, we have the following commutative diagram.

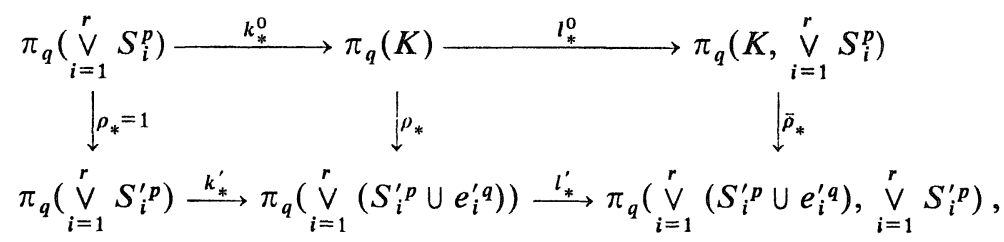


where $k^{0}, l^{0}, k^{\prime}$, and $l^{\prime}$ are inclusion maps and $\bar{\rho}$ is the relativization of $\rho$.

Let $j:\left(S_{i}^{p} \cup e_{i}^{q}, S_{i}^{p}\right) \rightarrow\left(\vee_{i=1}^{r}\left(S_{i}^{p} \cup e_{i}^{q}\right), \quad \vee_{i=1}^{r} S_{i}^{p}\right), \bar{m}:\left(S_{i}^{p} \cup e_{i}^{q}, S_{i}^{p}\right) \rightarrow(K$, $\left.\vee_{i=1}^{r} S_{i}^{p}\right)$ be inclusion maps and let $\bar{j}^{\prime}, \bar{m}^{\prime}$ be similar for $\left(S_{i}^{\prime p} \cup e_{i}^{\prime q}, S_{i}^{\prime p}\right)$. Let $\kappa_{q}^{i} \in \pi_{q}\left(S_{i}^{p} \cup e_{i}^{q}, S_{i}^{p}\right) \cong H_{q}\left(S_{i}^{p} \cup e_{i}^{q}, S_{i}^{p}\right)$ be the generator corresponding to $\left[e_{i}^{q}\right]$ and define $\kappa_{q}^{\prime i} \in \pi_{q}\left(S_{i}^{\prime p} \cup e_{i}^{\prime q}, S_{i}^{p}\right)$ similarly. Then, we have

$$
\bar{\rho}_{*} \bar{m}_{*}^{\prime}\left(\kappa_{q}^{\prime i}\right)=\bar{j}_{*}^{\prime} \kappa_{q}^{\prime i}, \quad \bar{\rho}_{*} \bar{m}_{*}\left(\kappa_{q}^{i}\right)=\bar{j}_{*}^{\prime} \kappa_{q}^{\prime i} .
$$

The former is clear since $\bar{\rho} \circ \bar{m}^{\prime}=\bar{j}^{\prime}$. The latter is known from the following commutative diagram including the factorization of $\bar{m}_{*}$.

$$
\begin{gathered}
\bar{m}_{*}: \pi_{q}\left(S_{i}^{p} \cup e_{i}^{q}, S_{i}^{p}\right) \stackrel{j_{*}}{\longrightarrow} \pi_{q}\left(\bigvee_{i=1}^{r}\left(S_{i}^{p} \cup e_{i}^{q}\right), \underset{i=1}{\bigvee} S_{i}^{p}\right) \longrightarrow \pi_{q}\left(K, \underset{i=1}{\vee} S_{i}^{p}\right) \\
\pi_{q}\left(S_{i}^{\prime p} \cup e_{i}^{\prime q}, S_{i}^{\prime p}\right) \stackrel{\bar{j}_{*}^{\prime}}{\longrightarrow} \pi_{q}\left(\bigvee_{i=1}^{r}\left(S_{i}^{\prime p} \cup e_{i}^{\prime q}\right), \underset{i=1}{\vee} S_{i}^{\prime p}\right),
\end{gathered}
$$

where $\bar{f}_{*}\left(\bar{j}_{*} \kappa_{q}^{i}\right)=\bar{j}_{*}^{\prime} \kappa_{q}^{\prime i}$ since $\bar{f}_{*}\left[e_{i}^{q}\right]=\left[e_{i}^{q}\right]$ by the assumption.

We apply the homomorphism $h_{*}: \pi_{q}\left(\vee_{i=1}^{r}\left(S_{A_{i}}^{p} \vee S_{A_{i}}^{q}\right)\right) \rightarrow \pi_{q}(K) . \quad l_{*}^{0} h_{*} c_{q}^{A_{i}}$ $=\bar{m}_{*} \kappa_{q}^{i}-\bar{m}_{*}^{\prime} \kappa_{q}^{\prime i}$ is clear from the definition of $h$. Hence, by (4.1) and (4.2),

$$
\begin{gathered}
l_{*}^{\prime}\left(\rho_{*} h_{*} \iota_{q}^{A_{i}}\right)=\bar{\rho}_{*}\left(l_{*}^{0} h_{*} \iota_{q}^{A_{i}}\right)=\bar{\rho}_{*}\left(\bar{m}_{*} \kappa_{q}^{i}-\bar{m}_{*}^{\prime} \kappa_{q}^{\prime i}\right) \\
=\bar{\rho}_{*} \bar{m}_{*} \kappa_{q}^{i}-\bar{\rho}_{*} \bar{m}_{*}^{\prime} \kappa_{q}^{\prime i}=\bar{\jmath}_{*}^{\prime} \kappa_{q}^{\prime i}-\bar{j}_{*}^{\prime} \kappa_{q}^{\prime i}=0 .
\end{gathered}
$$

So that,

$$
\rho_{*} h_{*} \ell_{q}^{A_{i}}=k_{*}^{\prime} \theta_{i}^{\prime} \quad \text { for some } \quad \theta_{i}^{\prime} \in \pi_{q}\left({\underset{j}{j=1}}_{j}^{r} S_{j}^{\prime p}\right) .
$$

Then, applying $\rho_{*}$ to (3.6) and by (4.3),

$$
\begin{aligned}
& \sum_{i=1}^{r} \rho_{*} \bar{\delta}_{i}-\sum_{i=1}^{r} \rho_{*} \bar{\delta}_{i}^{\prime}=\sum_{i=1}^{r} \rho_{*} h_{*}\left(c_{p}^{A_{i}} \circ \eta_{i}+\left[\ell_{q}^{A_{i}}, \iota_{p}^{A_{i}}\right]\right) \\
& \quad=\sum_{i=1}^{r}\left(\rho_{*} h_{*} \iota_{p}^{A_{i} \circ} \eta_{i}+\left[\rho_{*} h_{*} \iota_{q}^{A_{i}}, \rho_{*} h_{*} \iota_{p}^{A_{i}}\right]\right) \\
& =\sum_{i=1}^{r}\left(k_{*}^{\prime} \iota_{p}^{\prime i} \circ \eta_{i}+\left[k_{*}^{\prime} \theta_{i}^{\prime}, k_{*}^{\prime} c_{p}^{\prime}\right]\right)=\sum_{i=1}^{r} k_{*}^{\prime}\left(c_{p}^{\prime i} \circ \eta_{i}+\left[\theta_{i}^{\prime}, \iota_{p}^{\prime i}\right]\right) .
\end{aligned}
$$

On the other hand, let $\sigma \in \pi_{p+q}\left(B, \vee_{i=1}^{r}\left(S_{i}^{p} \cup e_{i}^{q}\right)\right), \sigma^{\prime} \in \pi_{p+q}\left(B^{\prime}, \vee_{i=1}^{r}\left(S_{i}^{\prime p} \cup e_{i}^{\prime q}\right)\right)$ be the orientation generators and let $\delta=\partial \sigma, \delta^{\prime}=\partial \sigma^{\prime}$. Since $f$ is of degree 1

$$
f_{*}^{0} \delta=f_{*}^{0} \partial \sigma=\partial f_{*} \sigma=\partial \sigma^{\prime}=\delta^{\prime},
$$

and therefore,

$$
\begin{aligned}
& \sum_{i=1}^{r} \rho_{*} \bar{\delta}_{i}-\sum_{i=1}^{r} \rho_{*} \bar{\delta}_{i}^{\prime}=\sum_{i=1}^{r} f_{*}^{0} \delta_{i}-\sum_{i=1}^{r} \delta_{i}^{\prime}=f_{*}^{0}\left(\sum_{i=1}^{r} \delta_{i}\right)-\sum_{i=1}^{r} \delta_{i}^{\prime} \\
& =f_{*}^{0} \delta-\delta^{\prime}=0 .
\end{aligned}
$$


Thus, we have

$$
\sum_{i=1}^{r} k_{*}^{\prime}\left(\iota_{p}^{\prime i} \circ \eta_{i}+\left[\theta_{i}^{\prime}, \iota_{p}^{\prime i}\right]\right)=0
$$

(i) Now, we assume that $2 p>q+1$. Then, $\pi_{q}\left(\vee_{i=1}^{r} S_{i}^{\prime p}\right) \cong \pi_{q}\left(S_{1}^{\prime p}\right) \oplus \cdots \oplus$ $\pi_{q}\left(S_{r}^{\prime p}\right)$ and we have the unique summation $\theta_{i}^{\prime}=\sum_{j=1}^{r} \theta_{i j}^{\prime}, \theta_{i j}^{\prime} \in \pi_{q}\left(S_{j}^{p}\right), j=1$, $2, \cdots, r$. Let $\theta_{i j}^{\prime}=c_{p}^{\prime j} \circ \theta_{i j}, \theta_{i j} \in \pi_{q}\left(S^{p}\right)$, for $j=1,2, \cdots, r$. Then,

$$
\begin{aligned}
\iota_{p}^{\prime i} \circ & \eta_{i}+\left[\theta_{i}^{\prime}, \iota_{p}^{\prime i}\right]=\iota_{p}^{\prime i} \circ \eta_{i}+\sum_{j=1}^{r}\left[\theta_{i j}^{\prime}, \iota_{p}^{\prime i}\right] \\
& =\iota_{p}^{\prime i} \circ \eta_{i}+\sum_{j=1}^{r}\left[\ell_{p}^{\prime j} \circ \theta_{i j}, \iota_{p}^{\prime i}\right] \\
& =\iota_{p}^{\prime i} \circ\left(\eta_{i}+\left[\theta_{i i}, \ell_{p}^{i}\right]\right)+\sum_{j \neq i}\left[\iota_{p}^{\prime j} \circ \theta_{i j}, \ell_{p}^{\prime i}\right],
\end{aligned}
$$

and by Barcus-Barratt [1] or G. W. Whitehead [15],

$$
\left[\iota_{p}^{\prime j} \circ \theta_{i j}, \iota_{p}^{\prime i}\right]=\left[\ell_{p}^{\prime j}, \iota_{p}^{\prime i}\right] \circ(-1)^{p+q} E^{p-1} \theta_{i j},
$$

where $\theta_{i j}, j=1,2, \cdots, r$, are the suspension elements. Hence, we have

$$
\iota_{p}^{\prime i} \circ \eta_{i}+\left[\theta_{i}^{\prime}, \iota_{p}^{\prime i}\right]=\iota_{p}^{\prime i} \circ\left(\eta_{i}+\left[\theta_{i i}, \iota_{p}^{i}\right]\right)+\sum_{j \neq i}\left[\iota_{p}^{\prime j}, \iota_{p}^{\prime i}\right] \circ(-1)^{p+q} E^{p-1} \theta_{i j} .
$$

Let $a_{i}=c_{p}^{\prime i_{\circ}}\left(\eta_{i}+\left[\theta_{i i}, c_{p}^{i}\right]\right), \beta_{j i}=(-1)^{p+q} E^{p-1} \theta_{i j}+(-1)^{q} E^{p-1} \theta_{j i}$, and $b_{j i}=\left[\iota_{p}^{\prime j}, \ell_{p}^{\prime i}\right]$ $\circ \beta_{j i}$, where $a_{i} \in \pi_{p+q-1}\left(S_{i}^{\prime p}\right) \subset \pi_{p+q-1}\left(\vee_{i=1}^{r} S_{i}^{\prime p}\right)$ and $b_{j i} \in \pi_{p+q-1}\left(S_{j}^{\prime p} \vee S_{i}^{\prime p}\right)$ $\subset \pi_{p+q-1}\left(\vee_{i=1}^{r} S_{i}^{p}\right), i, j=1,2, \cdots, r$. Then, by (4.4) and (4.5), we know

$$
\begin{aligned}
& \sum_{i=1}^{r} k_{*}^{\prime} a_{i}+\sum_{i<j} k_{*}^{\prime} b_{i j}=0, \quad \text { for } \quad k_{*}^{\prime}: \pi_{n}\left(\bigvee_{i=1}^{r} S_{i}^{\prime p}\right) \longrightarrow \pi_{n}\left(\bigvee_{i=1}^{r}\left(S_{i}^{\prime p} \cup e_{i}^{\prime q}\right)\right), \\
& n=p+q-1 .
\end{aligned}
$$

Here, each $k_{*}^{\prime} a_{i}$ belongs to the direct summand $\pi_{p+q-1}\left(S_{i}^{\prime p} \cup e_{i}^{q}\right)$. Now, assume temporarily that every $k_{*}^{\prime} b_{i j}$ belongs to another direct summand independent of $\pi_{p+q-1}\left(S_{i}^{\prime p} \cup e_{i}^{q}\right), i=1,2, \cdots, r$. This is the fact which will be shown in Assertion 3. Then, (4.6) yields $k_{*}^{\prime} a_{i}=0$ for $i=1,2, \cdots, r$, and by the commutative diagram

$$
\begin{aligned}
& \begin{array}{ccc}
\pi_{p+q-1}\left(S^{p}\right) \stackrel{k_{*}}{\longrightarrow} \pi_{p+q-1}\left(S^{p} \underset{\varepsilon_{i}^{\prime}}{\cup} e_{i}^{\prime q}\right) \\
\cong \ \ell_{p}^{\prime i} \quad & \cong \downarrow \mu_{*}
\end{array} \\
& \pi_{p+q-1}\left(S_{i}^{\prime p}\right) \stackrel{k_{*}^{\prime}}{\longrightarrow} \pi_{p+q-1}\left(S_{i}^{\prime p} \bigcup_{\ell_{p}^{\prime}{ }^{i} \varepsilon_{i}^{\prime}} e_{i}^{\prime q}\right),
\end{aligned}
$$

where $k_{*}$ is induced from the inclusion map and $\mu_{*}$ is the canonical isomorphism, we have 


$$
k_{*}\left(\eta_{i}+\left[\theta_{i i}, c_{p}\right]\right)=0, \quad i=1,2, \cdots, r,
$$

where $\eta_{i}=J \xi_{i}, \xi_{i} \in \pi_{q-1}\left(S O_{p}\right)$, and $i_{*} \breve{\zeta}_{i}=\alpha_{i}-\alpha_{i}^{\prime}, i=1,2, \cdots, r$. Here, $\left[\theta_{i i}, \iota_{p}\right]$ $=-J \partial \theta_{i i}, \partial: \pi_{q}\left(S^{p}\right) \rightarrow \pi_{q-1}\left(S O_{p}\right)$. Let $\xi_{i}^{\prime}=\xi_{i}-\partial \theta_{i i} \in \pi_{q-1}\left(S O_{p}\right)$. Then, $k_{*} J \xi_{i}^{\prime}$ $=k_{*}\left(J \xi_{i}-J \partial \theta_{i i}\right)=k_{*}\left(\eta_{i}+\left[\theta_{i i}, c_{p}\right]\right)=0, i=1,2, \cdots, r$. Since $\operatorname{Ker} k_{*}=\operatorname{Im}\left(\varepsilon_{i}^{\prime}\right)_{*}$, $\left(\varepsilon_{i}^{\prime}\right)_{*}=\varepsilon_{i}^{\prime \circ}: \pi_{p+q-1}\left(S^{q-1}\right) \rightarrow \pi_{p+q-1}\left(S^{p}\right)$ by (3.2) of James-Whitehead [10], J $\xi_{i}^{\prime}$ belongs to $\operatorname{Im}\left(\varepsilon_{i}^{\prime}\right)_{*}$, and $i_{*} \xi_{i}^{\prime}=i_{*}\left(\xi_{i}-\partial \theta_{i i}\right)=i_{*} \xi_{i}=\alpha_{i}-\alpha_{i}^{\prime}$. Hence, we know that $\alpha_{i}-\alpha_{i}^{\prime} \in i_{*}\left(J^{-1}\left(\operatorname{Im}\left(\varepsilon_{i}^{\prime}\right)_{*}\right)\right)=G\left(\varepsilon_{i}^{\prime}\right)$. That is, $\left\{\alpha_{i}\right\}=\left\{\alpha_{i}^{\prime}\right\}$ in $\pi_{q-1}\left(S O_{p+1}\right) / G\left(\varepsilon_{i}\right)$ $=\pi_{q-1}\left(S O_{p+1}\right) / G\left(\varepsilon_{i}^{\prime}\right), i=1,2, \cdots, r$.

(ii) Let $2 p=q+1(p, q>1)$. Then, $\pi_{q}\left(\vee_{i=1}^{r} S_{i}^{\prime p}\right)=\sum_{j=1}^{r} c_{p}^{\prime j} \circ \pi_{q}\left(S^{p}\right) \oplus$ $\sum_{j<k}\left[\iota_{p}^{\prime j}, \iota_{p}^{\prime k}\right] \circ \pi_{q}\left(S^{2 p-1}\right)$ by Hilton [4]. So that, we have the unique sum $\theta_{i}^{\prime}=\sum_{j=1}^{r} \iota_{p}^{\prime j} \circ \theta_{i j}+\sum_{j<k}\left[\iota_{p}^{\prime j}, \iota_{p}^{\prime k}\right] \circ \theta_{i j k}$, where $\theta_{i j} \in \pi_{q}\left(S^{p}\right), \theta_{i j k} \in \pi_{q}\left(S^{2 p-1}\right) \cong Z$ for any $i, j, k$. Therefore,

$$
\left[\theta_{i}^{\prime}, \iota_{p}^{\prime i}\right]=\sum_{j=1}^{r}\left[\iota_{p}^{\prime j} \circ \theta_{i j}, \iota_{p}^{\prime i}\right]+\sum_{j<k}\left[\left[\iota_{p}^{\prime j}, \iota_{p}^{\prime k}\right] \circ \theta_{i j k}, \iota_{p}^{\prime i}\right] .
$$

By (7.4) of Barcus-Barratt [1],

$$
\left[\iota_{p}^{\prime j} \circ \theta_{i j}, \iota_{p}^{\prime i}\right]=\left[\iota_{p}^{\prime j}, \iota_{p}^{\prime}\right] \circ(-1)^{p-1} E^{p-1} \theta_{i j}+\left[\iota_{p}^{\prime j},\left[\iota_{p}^{\prime j}, \iota_{p}^{\prime i}\right]\right] \circ(-1)^{p} E^{p-1} H_{0}\left(\theta_{i j}\right),
$$

where $H_{0}$ is the Hopf-Hilton homomorphism and the second term vanishes if $p$ is odd since $\theta_{i j}$ becomes a suspension element. And,

$$
\begin{aligned}
{\left[\left[c_{p}^{\prime j}, \iota_{p}^{\prime k}\right] \circ \theta_{i j k}, \iota_{p}^{\prime i}\right] } & =\left[\left[\iota_{p}^{\prime j}, c_{p}^{\prime k}\right], \iota_{p}^{\prime i}\right] \circ E^{p-1} \theta_{i j k} \\
& =\left[c_{p}^{\prime i},\left[c_{p}^{\prime j}, c_{p}^{\prime k}\right]\right] \circ(-1)^{p} E^{p-1} \theta_{i j k} .
\end{aligned}
$$

So that, we have

$$
\begin{aligned}
{\left[\theta_{i}^{\prime}, \iota_{p}^{\prime}\right]=\iota_{p}^{\prime i} \circ\left[\theta_{i i}, \iota_{p}^{i}\right] } & +\sum_{j \neq i}\left[\iota_{p}^{\prime j}, \iota_{p}^{\prime i}\right] \circ(-1)^{p-1} E^{p-1} \theta_{i j} \\
& +\sum_{j \neq i}\left[\iota_{p}^{j},\left[\iota_{p}^{\prime j}, \iota_{p}^{\prime i}\right]\right] \circ(-1)^{p} E^{p-1} H_{0}\left(\theta_{i j}\right) \\
& +\sum_{j<k}\left[\iota_{p}^{\prime i},\left[\iota_{p}^{\prime j}, \iota_{p}^{\prime k}\right]\right] \circ(-1)^{p} E^{p-1} \theta_{i j k} .
\end{aligned}
$$

Every Whitehead product of weight 3 is a linear combination of the Whitehead products $\left[\ell_{p}^{\prime i},\left[\iota_{p}^{\prime j}, \iota_{p}^{\prime k}\right]\right]$ such that $i \geqq j<k$ by using the Jacobi identity (Hilton [4]). Hence,

$$
\begin{aligned}
\sum_{i=1}^{r}\left(\iota_{p}^{\prime i} \circ \eta_{i}+\left[\theta_{i}^{\prime}, \iota_{p}^{\prime i}\right]\right)= & \sum_{i=1}^{r} \iota_{p}^{\prime i} \circ\left(\eta_{i}+\left[\theta_{i i}, \iota_{p}^{i}\right]\right)+\sum_{j<i}\left[\iota_{p}^{\prime j}, \iota_{p}^{\prime i}\right] \circ \beta_{j i} \\
& +\sum_{i \geqq j<k}\left[\iota_{p}^{\prime i},\left[\iota_{p}^{\prime j}, \iota_{p}^{\prime k}\right]\right] \circ \gamma_{i j k},
\end{aligned}
$$

where $\beta_{j i} \in \pi_{3 p-2}\left(S^{2 p-1}\right)(j<i)$ is defined as in (i) and $\gamma_{i j k}(i \geqq j<k)$ is a certain element of $\pi_{3 p-2}\left(S^{3 p-2}\right) \cong Z$. 
Let $a_{i}=c_{p}^{\prime i} \circ\left(\eta_{i}+\left[\theta_{i i}, c_{p}^{i}\right]\right), b_{j i}=\left[c_{p}^{\prime j}, c_{p}^{i}\right] \circ \beta_{j i}$ as in (i), and let $c_{i j k}=\left[c_{p}^{\prime i}\right.$, $\left.\left[\iota_{p}^{\prime j}, \iota_{p}^{\prime k}\right]\right] \circ \gamma_{i j k}$. Then, by (4.4) and (4.9), we know

$$
\sum_{i=1}^{r} k_{*}^{\prime} a_{i}+\sum_{i<j} k_{*}^{\prime} b_{i j}+\sum_{i \geqq j<k} k_{*}^{\prime} c_{i j k}=0
$$

where $k_{*}^{\prime}: \pi_{p+q-1}\left(\vee_{i=1}^{r} S_{i}^{\prime p}\right) \rightarrow \pi_{p+q-1}\left(\vee_{i=1}^{r}\left(S_{i}^{\prime p} \cup e_{i}^{\prime q}\right)\right), q=2 p-1$. Therefore, if we show that every $k_{*}^{\prime} b_{i j}$ and every $k_{*}^{\prime} c_{i j k}$ belong to the direct summands independent of $\pi_{p+q-1}\left(S_{i}^{\prime p} \cup e_{i}^{q}\right), i=1,2, \cdots, r$, then $k_{*}^{\prime} a_{i}=0$ for $i=1,2, \cdots, r$ by (4.10), and we can complete the proof similarly as in (i).

Thus, the following will conclude the proof of Assertion 2.

Assertion 3. Every $k_{*}^{\prime} b_{i j}(i<j)$ and every $k_{*}^{\prime} c_{i j k}(i \geqq j<k)$ are included in a direct summand of $\pi_{p+q-1}\left(\vee_{i=1}^{r}\left(S_{i}^{\prime p} \cup e_{i}^{q}\right)\right)$ which is independent of $\pi_{p+q-1}\left(S_{i}^{\prime p} \cup e_{i}^{q}\right), i=1,2, \cdots, r$.

Proof. Let $X_{t}=S_{t}^{\prime p} \cup e_{t}^{q q}, t=1,2, \cdots, r$. Then, $\pi_{n}\left(\vee_{t=1}^{r} X_{t}\right)=\sum_{t=1}^{r} \pi_{n}\left(X_{t}\right)$ $\oplus \partial \pi_{n+1}\left(\prod_{t=1}^{r} X_{t}, \vee_{t=1}^{r} X_{t}\right), n=p+q-1$. We have the following commutative diagram.

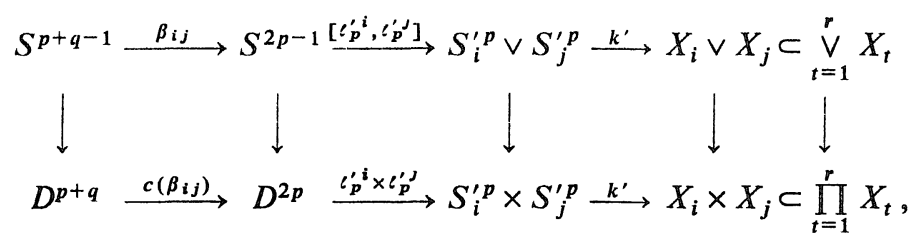

where vertical maps and $k^{\prime}$ are inclusions. Hence, $k_{*}^{\prime} b_{i j}$ belongs to $\partial \pi_{n+1}\left(\prod_{t=1}^{r} X_{t}, \vee_{t=1}^{r} X_{t}\right)$ which is independent of $\pi_{n}\left(X_{t}\right), t=1,2, \cdots, r$.

Generally, every basic product of weight $\geqq 2$ belongs to $\partial \pi_{n+1}\left(\prod_{t=1}^{r} S_{t}^{\prime p}\right.$, $\left.\vee_{t=1}^{r} S_{t}^{p}\right)$. In fact, in the splitting exact sequence

$$
\begin{aligned}
0 \longrightarrow \pi_{n+1}\left(\prod_{t=1}^{r} S_{t}^{\prime p}, \underset{t=1}{\bigvee_{t}} S_{t}^{\prime p}\right) \stackrel{\partial}{\longrightarrow} \pi_{n}\left(\bigvee_{t=1}^{r} S_{t}^{\prime p}\right) \\
\stackrel{i *}{\longrightarrow} \pi_{n}\left(\prod_{t=1}^{r} S_{t}^{\prime p}\right) \cong \sum_{t=1}^{r} \pi_{n}\left(S_{t}^{\prime p}\right) \longrightarrow 0,
\end{aligned}
$$

such Whitehead products are mapped to zero. So, for any basic product $\left[\iota_{p}^{\prime i},\left[\iota_{p}^{\prime j}, \iota_{p}^{\prime k}\right]\right](i \geqq j<k)$, there exists an element $\chi \in \pi_{n+1}\left(\prod_{t=1}^{r} S_{t}^{\prime p}, \vee_{t=1}^{r} S_{t}^{\prime p}\right)$ such that $\left[\iota_{p}^{\prime i},\left[\iota_{p}^{\prime j}, \iota_{p}^{\prime k}\right]\right]=\partial \chi$. Therefore, we have the following commutative diagram. 


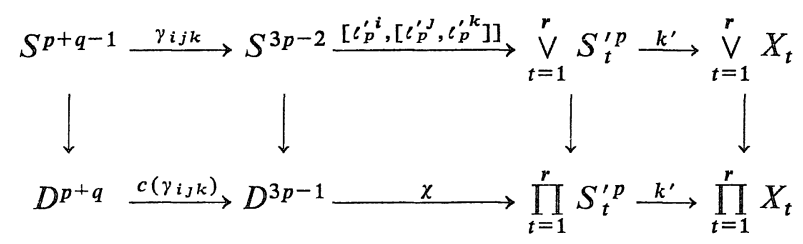

Hence, $k_{*}^{\prime} c_{i j k}$ belongs to $\partial \pi_{n+1}\left(\prod_{t=1}^{r} X_{t}, \vee_{i=1}^{r} X_{t}\right)$. This completes the proof.

Assertion 4. For "any" admissible basis $\left\{w_{1}^{\prime}, \cdots, w_{r}^{\prime}\right\}$ of $H^{\prime}=H_{q}\left(\#_{i=1}^{r} \bar{B}_{i}^{\prime}\right)$, there exists an admissible basis $\left\{w_{1}, \cdots, w_{r}\right\}$ of $H=H_{q}\left(\sharp_{i=1}^{r} \bar{B}_{i}\right)$ such that

(i) $\varepsilon\left(w_{i}\right)=\varepsilon^{\prime}\left(w_{i}^{\prime}\right), \quad i=1,2, \cdots, r$.

(ii) $\left\{\alpha\left(w_{i}\right)\right\}=\left\{\alpha^{\prime}\left(w_{i}^{\prime}\right)\right\}$ in $\pi_{q-1}\left(S O_{p+1}\right) / G\left(\varepsilon\left(w_{i}\right)\right)=\pi_{q-1}\left(S O_{p+1}\right) / G\left(\varepsilon^{\prime}\left(w_{i}^{\prime}\right)\right)$, $i=1,2, \cdots, r$.

Proof. There exists another expression of $\sharp_{i=1}^{r} B_{i}^{\prime}$ into a connected sum of $p$-sphere bundles over $q$-spheres $\#_{i=1}^{r} \widetilde{B}_{i}^{\prime}$ such that in the cell decomposition $\#_{i=1}^{r} \widetilde{B}_{i}^{\prime} \simeq\left\{\bigvee_{i=1}^{r}\left(\tilde{S}_{i}^{\prime p} \cup \tilde{e}_{i}^{\prime q}\right)\right\} \cup \tilde{e}^{\prime p+q}$, each homology class $\left[\tilde{e}_{i}^{\prime q}\right]$ corresponds to $w_{i}^{\prime}, i=1,2, \cdots, r$. Hence, Assertion 1 and Assertion 2 conclude the proof.

This completes the proof of the necessity for Theorem 4 .

\section{§5. Proof of the Sufficiency for Theorem 4}

Let $B_{i}, B_{i}^{\prime}$ be $p$-sphere bundles over $q$-spheres $(2 p>q>1)$ with the characteristic elements $\alpha_{i}, \alpha_{i}^{\prime}$ respectively and let $\varepsilon_{i}=\pi_{*}\left(\alpha_{i}\right), \varepsilon_{i}^{\prime}=\pi_{*}\left(\alpha_{i}^{\prime}\right)$, where $i=1$, $2, \cdots, r$. Let $\left\{w_{1}, \cdots, w_{r}\right\},\left\{w_{1}^{\prime}, \cdots, w_{r}^{\prime}\right\}$ be the admissible bases of $H, H^{\prime}$ respectively, satisfying

(i) $\varepsilon\left(w_{i}\right)=\varepsilon^{\prime}\left(w_{i}^{\prime}\right), \quad i=1,2, \cdots, r$, and

(ii) $\left\{\alpha\left(w_{i}\right)\right\}=\left\{\alpha^{\prime}\left(w_{i}^{\prime}\right)\right\}$ in $\pi_{q-1}\left(S O_{p+1}\right) / G\left(\varepsilon\left(w_{i}\right)\right)=\pi_{q-1}\left(S O_{p+1}\right) / G\left(\varepsilon^{\prime}\left(w_{i}^{\prime}\right)\right)$, $i=1,2, \cdots, r$.

By adopting the representations of the connected sums of given bundles using the admissible bases $\left\{w_{1}, \cdots, w_{r}\right\},\left\{w_{1}^{\prime}, \cdots, w_{r}^{\prime}\right\}$, we may assume that $w_{i}, w_{i}^{\prime}$ are represented by zero cross-sections of $\bar{B}_{i}, \bar{B}_{i}^{\prime}$ respectively, $i=1,2, \cdots, r$. Then, $\alpha\left(w_{i}\right)=\alpha_{i}, \alpha^{\prime}\left(w_{i}^{\prime}\right)=\alpha_{i}^{\prime}, \varepsilon\left(w_{i}\right)=\varepsilon_{i}$, and $\varepsilon^{\prime}\left(w_{i}^{\prime}\right)=\varepsilon_{i}^{\prime}, i=1,2, \cdots, r$. Hence, the proof is accomplished by directly extending that of James-Whitehead [10] ((1.5), p. 163).

Since $\alpha_{i}-\alpha_{i}^{\prime} \in G\left(\varepsilon_{i}\right)$, there exists an element $\xi_{i} \in \pi_{q-1}\left(S O_{p}\right)$ such that $i_{*} \xi_{i}$ $=\alpha_{i}-\alpha_{i}^{\prime}$ and $J \xi_{i} \in \operatorname{Im}\left(\varepsilon_{i}\right)_{*}, i=1,2, \cdots, r$. By (3.2) of [10], the sequence

$$
\pi_{p+q-1}\left(S^{q-1}\right) \stackrel{\left(\varepsilon_{i}\right)_{*}}{\longrightarrow} \pi_{p+q-1}\left(S^{p}\right) \stackrel{\left(k_{i}\right)_{*}}{\longrightarrow} \pi_{p+q-1}\left(S^{p} \bigcup_{\varepsilon_{i}} e^{q}\right)
$$


is exact, where $\left(k_{i}\right)_{*}$ is induced from the inclusion. Hence, $J \xi_{i} \in \operatorname{Im}\left(\varepsilon_{i}\right)_{*}$ $=\operatorname{Ker}\left(k_{i}\right)_{*}, i=1,2, \cdots, r$. Let $B_{i}=S_{i}^{p} \cup e_{i}^{q} \cup e_{i}^{p+q}, B_{i}^{\prime}=S_{i}^{\prime p} \cup e_{i}^{\prime q} \cup e_{i}^{p+q}$ be the cell-decompositions given by (3.3) of [9], where $e_{i}^{q}, e_{i}^{\prime q}$ are attached by $c_{p}^{i} \circ \varepsilon_{i}$, $\iota_{p}^{\prime}{ }^{i} \circ \varepsilon_{i}^{\prime}$ respectively. We identify $S^{p}$ canonically with $S_{i}^{p}, S_{i}^{\prime p}$ so that $\iota_{p}^{i}=\iota_{p}=\iota_{p}^{\prime i}$. Since $\varepsilon_{i}=\varepsilon_{i}^{\prime}$, there exists a homotopy equivalence $g_{i}: S^{p} \cup e_{i}^{q} \rightarrow S^{p} \cup e_{i}^{\prime q}$ such that $g_{i} \mid S^{p}=$ id. Let $\sigma_{i} \in \pi_{p+q}\left(B_{i}, S^{p} \cup e_{i}^{q}\right), \sigma_{i}^{\prime} \in \pi_{p+q}\left(B_{i}^{\prime}, S^{p} \cup e_{i}^{\prime q}\right)$ be the orientation generators and let $\delta_{i}=\partial \sigma_{i}, \delta_{i}^{\prime}=\partial \sigma_{i}^{\prime}$. Then, by (3.3) and Lemma (3.8) of [10],

(i) $\left(g_{i}\right)_{*} \delta_{i}-\delta_{i}^{\prime}=\left(k_{i}\right)_{*} J \xi_{i}^{\prime}$ for some $\xi_{i}^{\prime} \in \pi_{q-1}\left(S O_{p}\right)$ such that $i_{*} \xi_{i}^{\prime}=\alpha_{i}-\alpha_{i}^{\prime}$, and

(ii) $g_{i}$ can be chosen so that $\xi_{i}^{\prime}$ is a given element in $i_{*}^{-1}\left(\alpha_{i}-\alpha_{i}^{\prime}\right)$.

Hence, by taking $\xi_{i}$ as $\xi_{i}^{\prime}$, there exists a homotopy equivalence $g_{i}: S^{p} \cup e_{i}^{q}$ $\rightarrow S^{p} \cup e_{i}^{\prime q}$ such that $g_{i} \mid S^{p}=\mathrm{id}$ and $\left(g_{i}\right)_{*} \delta_{i}=\delta_{i}^{\prime}$, where $i=1,2, \cdots, r$.

In the cell-decompositions $\#_{i=1}^{r} B_{i} \simeq B=\left\{\vee_{i=1}^{r}\left(S_{i}^{p} \cup e_{i}^{q}\right)\right\} \cup e^{p+q}, \#_{i=1}^{r} B_{i}^{\prime} \simeq$ $B^{\prime}=\left\{\vee_{i=1}^{r}\left(S_{i}^{\prime p} \cup e_{i}^{\prime q}\right)\right\} \cup e^{p+q}, \quad$ let $\quad \sigma \in \pi_{p+q}\left(B, \vee{ }_{i=1}^{r}\left(S_{i}^{p} \cup e_{i}^{q}\right)\right), \sigma^{\prime} \in \pi_{p+q}\left(B^{\prime}\right.$, $\left.\vee_{i=1}^{r}\left(S_{i}^{\prime} \cup \cup e_{i}^{\prime q}\right)\right)$ be the orientation generators, and let $\delta=\partial \sigma, \delta^{\prime}=\partial \sigma^{\prime}$. Then,

$$
\delta=\delta_{1}+\delta_{2}+\cdots+\delta_{r}, \quad \delta^{\prime}=\delta_{1}^{\prime}+\delta_{2}^{\prime}+\cdots+\delta_{r}^{\prime},
$$

where it is understood that $\sum_{i=1}^{r} \pi_{p+q-1}\left(S_{i}^{p} \cup e_{i}^{q}\right) \subset \pi_{p+q-1}\left(\vee_{i=1}^{r}\left(S_{i}^{p} \cup e_{i}^{q}\right)\right)$ and $\sum_{i=1}^{r} \pi_{p+q-1}\left(S_{i}^{\prime p} \cup e_{i}^{\prime q}\right) \subset \pi_{p+q-1}\left(\vee_{i=1}^{r}\left(S_{i}^{\prime p} \cup e_{i}^{\prime q}\right)\right)$. Now, let

$$
g=\bigvee_{i=1}^{r} g_{i}: \bigvee_{i=1}^{r}\left(S_{i}^{p} \cup e_{i}^{q}\right) \longrightarrow \bigvee_{i=1}^{r}\left(S_{i}^{\prime p} \cup e_{i}^{\prime q}\right)
$$

Then, $g_{*} \delta=\sum_{i=1}^{r} g_{*} \delta_{i}=\sum_{i=1}^{r}\left(g_{i}\right)_{*} \delta_{i}=\sum_{i=1}^{r} \delta_{i}^{\prime}=\delta^{\prime}$, that is, $g_{*} \delta=\delta^{\prime}$. Hence, $g$ has an extension $f: B \rightarrow B^{\prime}$ of degree 1. $f_{*}: H_{n}(B) \rightarrow H_{n}\left(B^{\prime}\right)$ is isomorphic for $n=0, p, p+q$, and for $n=q$ as is shown by the following diagram

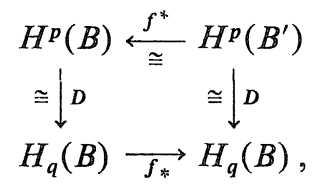

where $f_{*} \circ\left(D \circ f^{*} \circ D^{-1}\right)=\mathrm{id}$ and $D$ is the Poincaré duality isomorphism. Since $B, B^{\prime}$ are simply connected, $f$ is a homotopy equivalence. This completes the proof.

\section{References}

[1] Barcus, W. D. and Barratt, M. G., On the homotopy classification of the extensions of a fixed map, Trans. Amer. Math. Soc., 88 (1958), 57-74. 
[2] Boardman, J. M. and Steer, B., On Hopf invariants, Comment. Math. Helv., 42 (1967), 180-221.

[ 3 ] Haefliger, A., Differentiable links, Topology, 1 (1962), 241-244.

[4] Hilton, P. J., On the homotopy groups of the union of spheres, J. London Math. Soc., 30 (1955), 154-172.

[5] Ishimoto, H., Representing handlebodies by plumbing and surgery, Publ. RIMS. Kyoto Univ., 7 (1972), 483-510.

[6] - On the classification of $(n-2)$-connected $2 n$-manifolds with torsion free homology groups, ibid., 9 (1973), 211-260.

[7] — On the classification of some (n-3)-connected (2n-1)-manifolds, ibid., 11 (1976), 723-747.

[8] - Homotopy classification of connected sums of sphere bundles over spheres, I, Proc. Japan Acad., 55 (1979), 306-308, Nagoya Math. J., 83 (1981), to appear.

[9] James, I. M. and Whitehead, J. H. C., The homotopy theory of sphere bundles over spheres (I), Proc. London Math. Soc., (3) 4 (1954), 196-218.

[10] - The homotopy theory of sphere bundles over spheres (II), ibid., (3) 5 (1955), 148-166.

[11] Milnor, J., Lectures on the h-cobordism theorem, Princeton, 1965.

[12] Smale, S., On the structure of manifolds, Amer. J. Math., 84 (1962), 387-399.

[13] Tamura, I., On the classification of sufficiently connected manifolds, J. Math. Soc. Japan, 20 (1968), 371-389.

[14] Wall, C. T. C., Classification problems in differential topology - I, Classification of handlebodies, Topology, 2 (1963), 253-261.

[15] Whitehead, G. W., A generalization of the Hopf invariant, Ann. of Math., 51 (1950), 192-237.

[16] —_ Elements of homotopy theory, Springer-Verlag, 1978. 